\title{
Prediction and analysis of novel key genes ITGAX, LAPTM5, SERPINE1 in clear cell renal cell carcinoma through bioinformatics analysis
}

\author{
Yingli Sui ${ }^{1}$, Kun Lu ${ }^{1}$, Lin Fu ${ }^{\text {Corresp. } 1}$ \\ ${ }^{1}$ Institute of Chronic Disease, School of Basic Medicine, Qingdao University, Qingdao, Shandong, China \\ Corresponding Author: Lin Fu \\ Email address: fulin@qdu.edu.cn
}

Background: Clear Cell Renal Cell Carcinoma (CCRCC) is the most aggressive subtype of Renal Cell Carcinoma (RCC) with high metastasis and recurrence rates. This study aims to find new potential key genes of CCRCC. Methods: Four gene expression profiles 7GSE12606, GSE53000, GSE68417, and GSE66272) were downloaded from the Gene Expression Omnibus (GEO) database. The TCGA KIRC database was downloaded from The Cancer Genome Atlas (TCGA). Using GEO2R, the differentially expressed genes (DEG) in CCRCC tissues and normal samples were analyzed. Gene ontology (GO) and Kyoto Encyclopedia of Genes and Genomes (KEGG) enrichment analyses were performed in DAVID database. A protein-protein interaction (PPI) network was constructed and the hub gene was predicted by STRING and Cytoscape. GEPIA and Kaplan-Meier plotter databases were used for further screening of Key genes. Expression verification and survival analysis of key genes were performed using TCGA database, GEPIA database, and Kaplan-Meier plotter. Receiver operating characteristic (ROC) curve was used to analyze the diagnostic value of key genes in CCRCC, which is plotted by R software based on TCGA database. UALCAN database was used to analyze the relationship between key genes and clinical pathology in CCRCC and the methylation level of the promoter of key genes in CCRCC.Results: A total of 289 up-regulated and 449 down-regulated genes were identified based on GSE12606, GSE53000, GSE68417, and GSE66272 profiles in CCRCC. The upregulated DEGs were mainly enriched with protein binding and PI3K-Akt signaling pathway, whereas down-regulated genes were enriched with the integral component of the membrane and metabolic pathways. Next, top 35 genes were screened out from the PPI network according to Degree, and three new key genes ITGAX, LAPTM5 and SERPINE1 were further screened out through survival and prognosis analysis. Further results showed that the ITGAX, LAPTM5, and SERPINE1 levels in CCRCC tumor tissues were significantly higher than those in normal tissues and were associated with poor prognosis. ROC curve shows that ITGAX LAPTM5, and SERPINF1 have good diagnostic value with good specificity 
and sensitivity. The promoter methylation levels of ITGAX, LAPTM5 and SERPINE1 in CCRCC tumor tissues were significantly lower than those in normal tissues. We also found that key genes were associated with clinical pathology in CCRCC. Conclusion: ITGAX, LAPTM5, and SERPINE1 were identified as novel key candidate genes that could be used as prognostic biomarkers and potential therapeutic targets for CCRCC. 
2 Prediction and analysis of novel key Genes ITGAX, LAPTM5, SERPINE1 in

3 Clear Cell Renal Cell Carcinoma through bioinformatics analysis

4

6

\author{
Yingli Sui ${ }^{1}$, Kun $\mathrm{Lu}^{1}$, Lin $\mathrm{Fu}^{\text {corresp1 }}$ \\ ${ }^{1}$ Institute of Chronic Disease, School of Basic Medicine, Qingdao University, Qingdao, Shandong, China \\ Corresponding Author: Lin Fu Email address: fulin@qdu.edu.cn
}

\title{
Abstract
}

Background: Clear Cell Renal Cell Carcinoma (CCRCC) is the most aggressive subtype of Renal Cell Carcinoma (RCC) with high metastasis and recurrence rates. This study aims to find new potential key genes of CCRCC.

Methods: Four gene expression profiles (GSE12606, GSE53000, GSE68417, and GSE66272) were downloaded from the Gene Expression Omnibus (GEO) database. The TCGA KIRC database was downloaded from The Cancer Genome Atlas (TCGA). Using GEO2R, the differentially expressed genes (DEG) in CCRCC tissues and normal samples were analyzed. Gene ontology (GO) and Kyoto Encyclopedia of Genes and Genomes (KEGG) enrichment analyses were performed in DAVID database. A protein-protein interaction (PPI) network was constructed and the hub gene was predicted by STRING and Cytoscape. GEPIA and Kaplan-Meier plotter databases were used for further screening of Key genes. Expression verification and survival analysis of key genes were performed using TCGA database, GEPIA database, and Kaplan-Meier plotter. Receiver operating characteristic (ROC) curve was used to analyze the diagnostic value of key genes in CCRCC, which is plotted by R software based on TCGA database. UALCAN database was used to analyze the relationship between key genes and clinical pathology in CCRCC and the methylation level of the promoter of key genes in CCRCC.

Results: A total of 289 up-regulated and 449 down-regulated genes were identified based on GSE12606, GSE53000, GSE68417, and GSE66272 profiles in CCRCC. The upregulated DEGs were mainly enriched with protein binding and PI3K-Akt signaling pathway, whereas downregulated genes were enriched with the integral component of the membrane and metabolic pathways. Next, top 35 genes were screened out from the PPI network according to Degree, and three new key genes ITGAX, LAPTM5 and SERPINE1 were further screened out through survival and prognosis analysis. Further results showed that the ITGAX, LAPTM5, and SERPINE1 levels in CCRCC tumor tissues were significantly higher than those in normal tissues and were associated with poor prognosis. ROC curve shows that ITGAX, LAPTM5, and SERPINE1 have good diagnostic value with good specificity and sensitivity. The promoter methylation levels of ITGAX, 
LAPTM5 and SERPINE1 in CCRCC tumor tissues were significantly lower than those in normal tissues. We also found that key genes were associated with clinical pathology in CCRCC.

Conclusion: ITGAX, LAPTM5, and SERPINE1 were identified as novel key candidate genes that could be used as prognostic biomarkers and potential therapeutic targets for CCRCC.

Keywords: protein-protein interaction; CCRCC; bioinformatical analysis; differentially

expressed genes.

\section{Introduction}

Kidney cancer is a complex disease composed of a variety of cancers, showing different histology, clinical course, genetic changes, and response to treatment ${ }^{1}$. Renal Cell Carcinoma is the most common tumor in the kidney, Whose morbidity and mortality are rising worldwide. Renal cell carcinoma is divided into different subtypes, including clear cell renal cell carcinoma (CCRCC), chromogenic cell renal carcinoma (chRCC), and papillary renal cell carcinoma (pRCC) ${ }^{2}$. CCRCC is a metabolic disease ${ }^{3}$, accounting for more than $80 \%$ of all renal cell carcinomas ${ }^{4}$. It is the most aggressive subtype of renal cell carcinoma with a high rate of metastasis and recurrence ${ }^{5,}{ }^{6}$. Although some progress has been made in the treatment of CCRCC, the current treatments of CCRCC still focus on surgical treatment and traditional chemotherapy ${ }^{7,8}$. At the same time, there is a lack of effective early diagnosis methods in the clinic, and some patients still have a relapse and targeted drug tolerance, leading to poor prognosis of radiotherapy and chemotherapy. Therefore, finding new targeted biomarkers relevant to the diagnosis and treatment of CCRCC remains of paramount importance.

In this study, bioinformatics methods were used to obtain CCRCC gene expression data from GEO database, and normal samples and CCRCC samples were selected for grouping processing. Next, 738 DEGs were screened, including 289 up-regulated genes and 449 down-regulated genes. And then, GO enrichment analysis and KEGG signal pathway analysis were performed by DAVID. The up-regulated DEGs are mainly concentrated on protein binding, plasma membrane, inflammation, signal transduction, and PI3K-Akt signaling pathways, while the down-regulated genes are mainly concentrated on the extracellular exosome, oxidation-reduction process, integral component of membrane, protein homodimerization activity, and metabolic pathways. Finally, the top 35 hub genes were screened by PPI network. Based on the novelty of ITGAX, LAPTM5 and SERPINE1 that have not been reported in CCRCC, the expression of these three genes is significantly associated with survival prognosis and all have high degrees. Therefore, ITGAX, LAPTM5, and SERPINE1 were finally selected as key genes. Further analysis showed that 
70 ITGAX, LAPTM5, and SERPINE1 are highly expressed in CCRCC, which are significantly 71 related to the survival prognosis of CCRCC. The methylation level of ITGAX, LAPTM5, 72 SERPINE1 in CCRCC is reduced. Moreover, ITGAX, LAPTM5 and SERPINE1 are related to 73 the Clinical pathology of CCRCC and have good diagnostic value for CCRCC. In conclusion, we 74 provided a systematic and comprehensive analysis of CCRCC and is the first to suggest that ITGAX, LAPTM5, and SERPINE1 might be used as biomarkers for the new clinical diagnosis and treatment of CCRCC.

77

78

79

80

81

82

83

84

85

86

87

88

89

90

91

92

93

94

95

96

97

98

99

100

101

102

103

104

105

106

\section{Materials \& Methods}

Data collection. Four gene expression profiles (GSE12606, GSE53000, GSE68417, and GSE66272) were downloaded from the $\mathrm{GEO}^{9}$ database (http://www.ncbi.nlm. nih.gov/geo/). The TCGA KIRC database was downloaded from TCGA (https:/www.cancer.gov/about-nci /organization /ccg/research/structural-genomics/tcga). The GSE12606 ${ }^{10}$ data sets was obtained by GPL570 platform, including three adjacent normal kidney specimens, and three CCRCC samples. The GSE53000 11 and GSE68417 12 data sets were based on GPL6244 Platforms. GSE53000 data sets was comprised of 60 samples including 6 adjacent normal kidney specimens and 54 CCRCC samples and GSE68417 data sets was comprised of 43 samples including 14 adjacent normal kidney specimens and 29 CCRCC samples. GSE66272 $2^{13}, 14$ data sets was obtained by GPL5029 platform and contained 27 CCRCC tumor samples and 26 normal kidney samples. The TCGA KIRC database contained 539 CCRCC tumor samples and 72 normal kidney samples.

Study design and data processing. In order to clarify our study, we designed a flow chart to demonstrate data collection, processing, analysis and verification (FIG 1). The online tool GEO2R (http:/www.ncbi.nlm.nih.gCCRCC/geo/geo2r) is an online analysis of GEO series based on the $\mathrm{R}$ programming language, which is used to screen DEGs between normal kidney and CCRCC samples from the GSE data sets. The data was standardized and filtered to select significant DEGs. P-value $<0.05, \log$ Fold Change $(|\log \mathrm{FC}|) \geqslant 1$ were chosen as the cutoff criteria. log Fold Change $(|\log \mathrm{FC}|) \geqslant 1$ means that the multiple of change is greater than or equal to 2 . It is generally considered that there is a difference between 2 times and more. Then, DEGs were further screened according to cutoff criteria: $\mathrm{P}$-value $<0.05, \log \mathrm{FC} \geqslant 1$ as up-regulated genes, $\mathrm{P}$-value $<0.05, \log$ $\mathrm{FC} \leqslant 1$ as down-regulated genes. Finally, importing all the up-regulated genes or down-regulated genes in the 4 datasets into Funrich 3.1.1 software, and taking the intersection of the up-regulated genes or down-regulated genes in the 4 datasets respectively. TCGA RNA-seq simple converter was used to standardize and $\log 2$ conversion of TCGA KIRC data. TCGA KIRC data were used for expression verification and ROC curve analysis of Key genes.

Enrichment analysis of DEGs. DAVID 6.8 (Database for Annotation, Visualization and Integrated Discovery, https://david.ncifcrf.gov/) ${ }^{15,16}$ database was used to analyze Gene Ontology $(\mathrm{GO})^{17}$ such as the biological process $(\mathrm{BP})^{18}$, cellular component $(\mathrm{CC})^{19}$ and molecular function (MF), and Kyoto Encyclopedia of Genes and Genomes (KEGG) ${ }^{20}$ pathway enrichment analysis of 
107 important DEGs, which promoted the visualization of gene and protein function ${ }^{21}$. Among them, 108 the count value represents the number of genes enriched in the pathway. The cutoff value was $109 \quad \mathrm{P}<0.05$.

110 Protein-Protein Interaction (PPI) Network construction and hub gene screening. The online

111

112

113

114

115

116

117

118

119

120

121

122

123

124

125

126

127

128

129

130

131

132

133

134

135

136

137

138

139

140

141

142

143

144

145

146 tool STRING ${ }^{22}$ (http://string-db.org) was utilized for the analysis of protein-protein interactions . Importing all DEGs (including up-regulated genes and down-regulated genes) into the STRING database for analysis, and the confidence level $\geqslant 0.4$ is considered to be significant for PPI. STRING and Cytoscape software (version 3.6.1) were used to construct the PPI network, which is regarded as a network software platform for visualizing protein molecular interactions. Using the degree algorithm in the cytohubba plug-in of Cytoscape, the degree value could be calculated and thus the hub genes could also be screened from PPI network. The importance of genes is directly related to the degree of protein. According to the degree value (degree $\geqslant 37$ ), the first 35 genes were selected as hub genes. Three key genes ITGAX, LAPTM5 and SERPINE1with high degree were screened through survival and prognosis analysis as well as the novelty of genes.

Key genes analysis and verification. Three key genes were analyzed and verified comprehensively by using TCGA, GEPIA, Oncomine, Kaplan-Meier plotter and UALCAN databases. Similarly, TCGA database also applied to ROC curve analysis. ROC curve was plotted by R software, which could be used to analyze the diagnostic value of key genes in CCRCC. GEPIA (http://gepia.cancerpku.cn/) analyzed the expression of key genes in CCRCC. Oncomine 23 (http://www.on comine.org) was a database consisting of microarray data of various tumors, which verified the expression of key genes in CCRCC. Kaplan-Meier Plotter (http://kmplot.com/analysis/index) was used to analyze the survival of key genes in CCRCC. UALCAN ${ }^{24}$ (http://ualcan.path. uab.edu/index.html) online database was used to analyze the relationship between key genes and Clinical pathology in CCRCC, and the promoter methylation level of key genes in CCRCC was analyzed.

Statistical analysis. Statistically significant differences between the normal tissues group and tumor tissues group were determined using Student's t tests. Also, Kaplan-Meier analysis was used to assess OS. ROC curve is a graphical plot ${ }^{25}$ that reflects the sensitivity and specificity of continuous variables. $\mathrm{P}<0.05$ was considered to be statistically significant for all the tests. All the statistical analyses applied GraphPad prism 6.0 or R software.

\section{Results}

Volcano plots of the differentially expressed genes in four datasets. The data sets of CCRCC, which were GSE12606, GSE53000, GSE68417 and GSE66272, were downloaded from the GEO database (Table 1) and analyzed by GEO2R separately. A total of 9,560 DEGs were screened from the GSE12606 data sets, among which 1568 genes were up-regulated and 5436 genes were downregulated instead. A total of 1,286 DEGs were screened from the GSE53000 data sets, among which 583 were up-regulated and 708 were down-regulated. A total of 1,890 DEGs were screened from the GSE68417 data sets among them 726 up-regulated and 1,164 down-regulated genes were selected. There were 3,627 up-regulated genes and 5,170 down-regulated genes among the 8,797 DEGs screened from the GSE66272 data sets. The screening criteria were P-value $<0.05, \log$ Fold 
147 Change $(|\log \mathrm{FC}|) \geqslant 1$. All of the DEGs from the four data sets were presented in Volcano plots

148 (FIG 2A-D). Among them, red represents high-expressed genes, green represents low-expressed 149 genes, and black represents genes whose expression levels are not significant in each data set. We gained the intersection of four independent data sets through Funrich 3.1.1 software and made a visualization analysis of Venn Diagram. After being overlapped, the common 738 genes (supplementary FIG.1) were identified, including 289 up-regulated and 449 down-regulated genes (FIG 2E-F).

GO and KEGG enrichment analysis of DEGs. To further explore the function of DEGs, enrichment analysis of up-regulated genes and down-regulated genes were displayed respectively. DAVID 6.8 was used to perform GO and KEGG analysis of DEGs in CCRCC (Table 2). In biological processes, up-regulated DEGs are mostly involved in cell adhesion, signal transduction, immune response, especially the regulation of inflammatory response (FIG 3A); while downregulated DEGs are mostly involved in oxidation-reduction process proteolysis, ion transmembrane transport, response to drug, and ion transport (FIG 3E). In terms of cellular components, up-regulated DEGs are mainly distributed in the plasma membrane, integral component of the membrane, extracellular exosome, and membrane (FIG 3B); down-regulated DEGs are mainly distributed in integral component of membrane, plasma membrane, cellular exosomes, and integral component of plasma membrane (FIG 3F). In terms of molecular function, up-regulated DEGs generally have protein binding, identical protein bind, ATP binding capacity, and protein homodimerization activity (FIG 3C); down-regulated DEGs generally have identical protein homodimerization activity, calcium ion binding, sequence-specific DNA binding, and oxidoreductase activity (FIG 3G). In the KEGG signal pathway, the up-regulation of DEGs mostly involved in the PI3K-Akt signaling pathway, pathways in cancer, focal adhesion, and HIF-1 signaling pathway (FIG 3D); while the down-regulation of DEGs mainly involved in the metabolic pathway, Biosynthesis of antibiotics, Carbon metabolism, and Aldosterone-regulated sodium reabsorption (FIG 3H). CCRCC is a kind of metabolic disease. Metabolic reprogramming covers different processes including aerobic glycolysis, fatty acid metabolism and the utilization of tryptophan, glutamine as well as arginine ${ }^{26}$, which has also been proved by the results of KEGG pathway enrichment analysis in the work. KEGG pathway results show that down-regulated DEGs are enriched in metabolic pathways, Glycolysis/Gluconeogenesis, Glycine, serine and threonine metabolism and so on. down-regulated genes) (supplementary FIG.2). Finally, 11 common enrichment results were found from the three analyses (including up-regulated DEG, down-regulated DEG and all DEG enrichment analyses). The common enrichment results are as follows: response to drug, plasma membrane, integral component of membrane, extracellular space, extracellular exosome, cell surface, cell surface, protein homodimerization activity, identical protein binding. The results of may be important in CCRCC. 
186

187

188

189

190

191

192

193

194

195

196

197

198

199

200

201

202

203

204

205

206

207

208

209

210

211

212

213

214

215

216

217

218

219

220

221

222

223

224

225

Construction of PPI network and screening of key genes. To identify the key genes, the STRING online database and Cytoscape software were used to analyze all DEGs (including up-regulated genes and down-regulated genes)and construct PPI network (FIG 4A). Based on the main role of proteins in biological functions, their interaction determines the molecular and cellular mechanisms that control the health and disease state of the organism ${ }^{29}$. Next, using the degree algorithm in the cytohubba plug-in of Cytoscape to screen the hub genes in PPI network. The gene whose degree $\geqslant 37$ could be defined hub gene, therefore the first 35 genes (Table 3 ) in the PPI network were chosen to be hub genes (FIG 4D). Next, GO enrichment analysis was conducted for all DEGs and the first 35 DEGs in the PPI network. The result showed that all DEGs mainly enriched in signal transduction, oxidation-reduction process, cell adhesion, inflammatory response, plasma membrane, integral component of membrane, extracellular exosome, integral component of plasma membrane (FIG 4B-C). The top 35 DEGs were enriched in extracellular space, extracellular exosome, inflammatory response, an integral component of the plasma membrane, and cell surface (FIG 4E). By comparison, it turned out that the enrichment analysis results of all DEGs in PPI network contained the results of the top 35's. In the end, three new key genes with high degree which were ITGAX, LAPTM5 and SERPINE1, could be screened by using GEPIA and Kaplan-Meier plotter database.

The expression of key genes. Among the 35 genes, we focused on ITGAX, LAPTM5, and SERPINE1, which have not been reported to be related to the occurrence and development of CCRCC. Firstly, the expression levels of ITGAX, LAPTM5, and SERPINE1 in CCRCC tumor tissues are significantly higher than those in normal tissues adjacent to cancer according to GEPIA database (FIG 5A-C). Furthermore, the data of TCGA KIRC showed that compared with adjacent normal tissues, the mRNA expressions of ITGAX, LAPTM5, and SERPINE1 in 72 pairs of CCRCC tissues were significantly increased (FIG 5D-F). In the Oncomine Gumz renal database, the mRNA levels of ITGAX, LAPTM5 and SERPINE1 were also upregulated in CCRCC tissues when compared with adjacent normal kidney tissues (FIG 5H-G). According to the UALCAN database, the promoter methylation levels of ITGAX, LAPTM5 and SERPINE were decreased in CCRCC (FIG 5J-L). To sum up, according to the GEPIA database, Oncomine Gumz renal database and TCGA database, the expression levels of these three genes in CCRCC tumor tissues are significantly higher than those in normal tissues adjacent to cancer. It could be further speculated that the high expression of ITGAX, LAPTM5, and SERPINE1 in CCRCC tumor tissue might be related to the decrease of promoter methylation.

\section{The association of key genes expression with clinical pathology in CCRCC.}

Furthermore, the relationship between the mRNA expression of ITGAX, LAPTM5 and SERPINE1 and different clinical pathology grades were measured. The results showed that their mRNA expression was significantly related to pathological grades (FIG 6A-C). And the expression of ITGAX, LAPTM5, and SERPINE1 mRNA in CCRCC samples are also significantly correlated with severe clinical staging (FIG6 D-F). Among them, the expression levels of ITGAX, LAPTM5, and SERPINE1 were higher in stage 4 and grade 4. In conclusion, ITGAX, LAPTM5, and SERPINE1 are significantly associated with clinical pathology. 
226

227

228

229

230

231

232

233

234

235

236

237

238

239

240

241

242

243

244

245

246

247

248

249

250

251

252

253

254

255

256

257

258

259

260

261

262

263

264

Survival and diagnostic value of ITGAX, LAPTM5, and SERPINE1 in CCRCC. According to the Kaplan-Meier plotter databases, the overall survival of ITGAX, LAPTM5, and SERPINE1 genes was tested (FIG 7A-C). The results showed that the high expression of three key genes in CCRCC was negatively correlated with prognosis. Then, the ROC curve was used to evaluate the difference between CCRCC and the normal tissues in the TCGA datasets. The ROC curves (FIG 7D-E) of these three genes showed that their area under the curve (AUC) and the $95 \%$ confidence intervals (CI) are as follows: ITGAX (AUC=96.315, CI $=0.9423-0.9840)$, LAPTM5, (AUC $=94.808 \mathrm{CI}=0.9243-0.9719$ ), and SERPINE1(AUC=76.272, CI:0.7068 to 0.8186). Since ROC curves had good specificity and sensitivity, ITGAX, LAPTM5, and SERPINE1 had excellent diagnostic efficiency for distinguishing tumors and normal tissues.

\section{Discussion}

Clear Cell Renal Cell Carcinoma (CCRCC) is a metabolic disease whose morbidity is rising worldwide. The feature of kidney cancer is to participate in the target genes' mutation of metabolic pathways ${ }^{26}$. Recently, with the application of bioinformatics, the molecular characteristics of CCRCC have been greatly improved and the development of targeted therapy has been promoted. These advances have significantly improved the median survival of patients with advanced disease. However, about $30 \%$ of CCRCC local patients will still relapse or metastasize after surgical removal of the tumor ${ }^{30}$. Around $1 / 3$ of the metastatic patients had poor prognosis and rare high drug resistance rate. Under the circumstance of different treatment, the identification of biomarkers was needed urgently so as to predict the drug's effects ${ }^{31}$. Therefore, identification of CCRCC key genes and prognostic judgment is still very crucial. In our research, some reported genes related to CCRCC, such as VEGFA ${ }^{32}$, EGFR ${ }^{33}$, were also screened out. Vascular Endothelial Growth Factor A (VEGFA) is a member of the PDGF/VEGF growth factor family. VEGFA has a potential role in the diagnosis and treatment of CCRCC. VEGFA can inhibit the proliferation of CCRCC 786-O cells, promote cell apoptosis, and inhibit cell migration and invasion ${ }^{32}$. Epidermal growth factor receptor (EGFR) is closely related to the progression of many epithelial malignancies and is an important therapeutic target ${ }^{33}$. EGFR is a cell surface protein, belonging to the ERBB family. EGFR binds to epidermal growth factor to induce receptor dimerization and tyrosine self phosphorylation, which eventually leads to cell proliferation ${ }^{34}$. EGFR can activate a variety of signaling pathways, mainly MAPK / ERK and PI3K / AKT pathways ${ }^{35}$. Compared with the former 4 studies, this study found three new key genes which were ITGAX, LAPTM5 and SERPINE1, and applied several methods to do functional analysis and systematic research to key genes such as methylation level analysis, survival analysis, ROC curve analysis and so on. In previous studies, GSE12606 mainly focused on the functional analysis of HLA ligand in CCRCC, while GSE5300 mainly focused on the structure and revolution of CCRCC genome, and GSE66272 mainly focused on the role of miRNA in CCRCC and GSE68417 mainly focused on the analysis to the gene expression profile of CCRCC. On the contrary, this study put emphasis on screening the biomarkers used to do early diagnosis in CCRCC and analyzing the intersection of DEGs in four datasets. 
265

266

267

268

269

270

271

272

273

274

275

276

277

278

279

280

281

282

283

284

285

286

287

288

289

290

291

292

293

294

295

296

297

298

299

300

301

302

303

304

305

306

Besides, three new potential marker genes ITGAX, LAPTM5, SERPINE1 were also screened out and proved. Integrin alpha $\mathrm{x}$ (ITGAX) is a member of the integrin family, commonly function as a receptor for extracellular matrix. It is reported that ITGAX is involved in the angiogenesis of dendritic cells and tumor angiogenesis ${ }^{36}$. In addition, ITGAX is identified as a new type of aggressive prostate cancer susceptibility gene ${ }^{37}$. Lysosomal protein transmembrane 5 (LAPTM5), known as E3 protein, may play a role in hematopoiesis and prevent excessive activation of lymphocytes ${ }^{38}$. It is reported that LAPTM5 can regulate the proliferation and viability of bladder cancer cells, leading to cell cycle arrest in the G0/G1 phase ${ }^{39}$. LAPTM5 is also associated with the spontaneous regression of neuroblastoma ${ }^{40}$. Studies have shown that Serpin family E member 1 (SERPINE1) is a regulator of Glioblastoma (GBM) cell proliferation, and is related to poor prognosis of patients and mesenchymal GBM. Down-regulation of SERPINE1 in primary GBM cells inhibited the growth and invasiveness of tumors in the brain, and SERPINE1 plays a key role in the spread of $\mathrm{GBM}^{41}$.

\section{Conclusions}

To sum up, the expression levels of ITGAX, LAPTM5, SERPINE1 in CCRCC tumor tissues are significantly higher than those in normal tissues adjacent to cancer and are related to the tumor stage and tumor grade. ITGAX, LAPTM5, and SERPINE1 have high diagnostic efficiency for tumors and normal tissues, and their expressions are associated with poor prognosis of CCRCC. The decrease of promoter methylation of ITGAX, LAPTM5 and SERPINE1 in CCRCC tumor tissues indicates that the high expression of key genes in CCRCC might be relevant to the low methylation level. The limitation of this study lied in that the internal molecular mechanisms where key genes played a role remained unclear, which need a further research. Further studies are needed to explore the detailed mechanisms of these key genes in CCRCC. In conclusion, we identified ITGAX, LAPTM5, and SERPINE1 as potential marker genes of CCRCC by bioinformatics methods, providing insights for future therapeutic design. Meanwhile, we conducted a relatively systematic and comprehensive analysis on CCRCC data, thereby providing a theoretical basis for identifying therapeutic targets of CCRCC, promoting early detection, and monitoring tumor progression.

\section{Acknowledgements}

This work was supported by grants from the National Natural Science Foundation of China 327 [81702743] and China Postdoctoral Science Foundation 326 [2018M640612, 2019T120568].

\section{References}

1. Linehan, W.M. et al. The Metabolic Basis of Kidney Cancer. Cancer discovery 9, 1006-1021 (2019).

2. Linehan, W.M. \& Ricketts, C.J. The Cancer Genome Atlas of renal cell carcinoma: findings and clinical implications. Nature reviews. Urology 16, 539-552 (2019).

3. Wettersten, H.I., Aboud, O.A., Lara, P.N., Jr. \& Weiss, R.H. Metabolic reprogramming in clear cell renal cell carcinoma. Nature reviews. Nephrology 13, 410-419 (2017).

4. Makhov, P. et al. Resistance to Systemic Therapies in Clear Cell Renal Cell Carcinoma: Mechanisms and Management Strategies. Molecular cancer therapeutics 17, 1355-1364 (2018).

5. Jiang, Y. et al. FOXM1-Activated LINC01094 Promotes Clear Cell Renal Cell Carcinoma Development via MicroRNA 224-5p/CHSY1. Molecular and cellular biology 40 (2020).

6. Yuan, L. et al. Identification of key genes and pathways in human clear cell renal cell carcinoma (ccRCC)

Peer) reviewing PDF | (2020:11:55180:1:0:NEW 16 Feb 2021) 


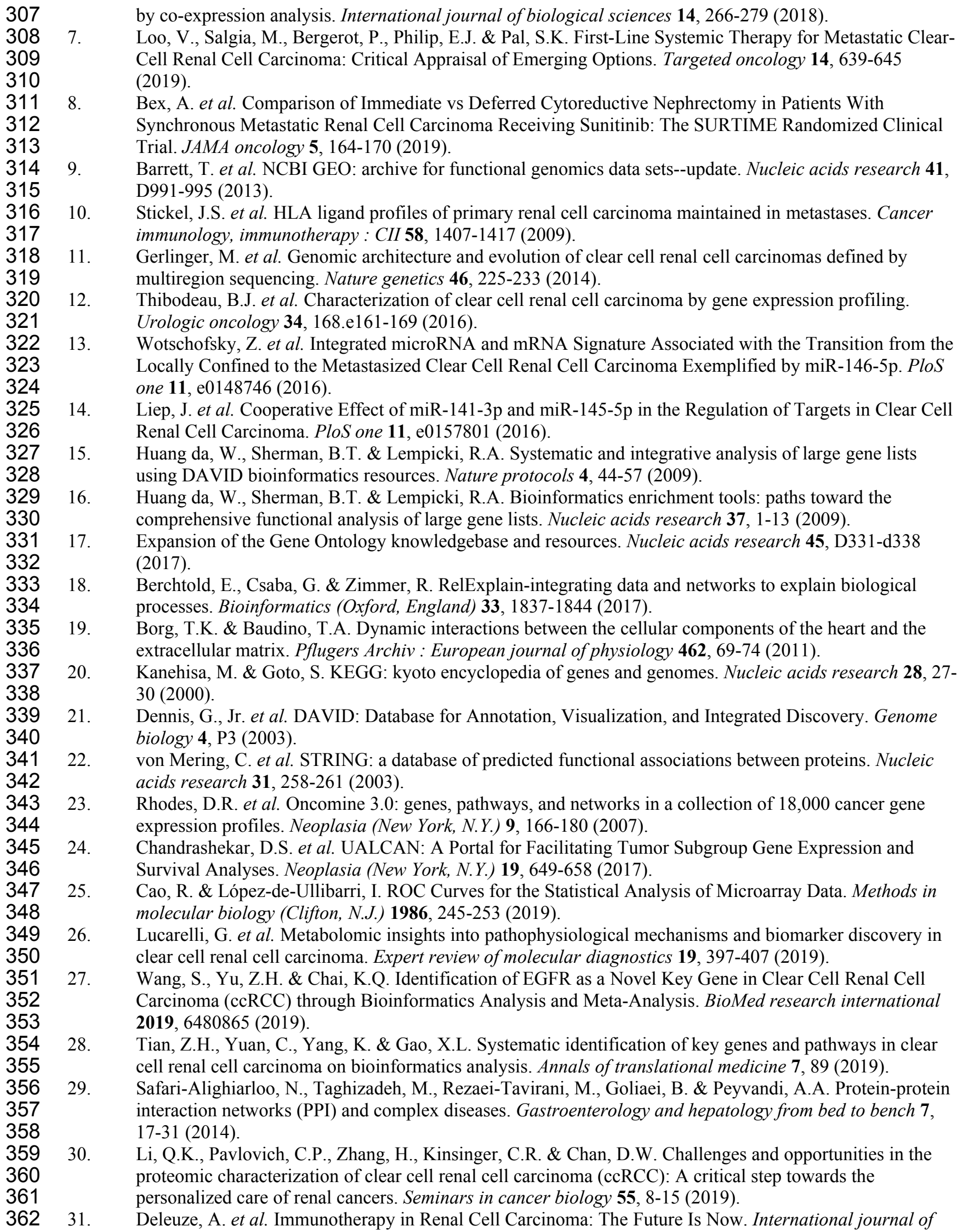


molecular sciences 21 (2020).

32. Zeng, F.C. et al. Downregulation of VEGFA inhibits proliferation, promotes apoptosis, and suppresses migration and invasion of renal clear cell carcinoma. OncoTargets and therapy 9, 2131-2141 (2016).

33. Cossu-Rocca, P. et al. EGFR kinase-dependent and kinase-independent roles in clear cell renal cell carcinoma. American journal of cancer research 6, 71-83 (2016).

34. Mitsudomi, T. \& Yatabe, Y. Epidermal growth factor receptor in relation to tumor development: EGFR gene and cancer. The FEBS journal 277, 301-308 (2010).

35. Yarden, Y.\& Sliwkowski, M.X. Untangling the ErbB signalling network. Nature reviews. Molecular cell biology 2, 127-137 (2001).

36. Wang, J., Yang, L., Liang, F., Chen, Y. \& Yang, G. Integrin alpha x stimulates cancer angiogenesis through PI3K/Akt signaling-mediated VEGFR2/VEGF-A overexpression in blood vessel endothelial cells. Journal of cellular biochemistry 120, 1807-1818 (2019).

37. Williams, K.A. et al. A systems genetics approach identifies CXCL14, ITGAX, and LPCAT2 as novel aggressive prostate cancer susceptibility genes. PLoS genetics 10, e1004809 (2014).

38. Cai, X. et al. Expression and Polymorphisms of Lysosome-Associated Protein Transmembrane 5 (LAPTM5) in Patients with Systemic Lupus Erythematosus in a Chinese Population. Biochemical genetics 53, 200-210 (2015).

39. Chen, L. et al. Downregulation of LAPTM5 suppresses cell proliferation and viability inducing cell cycle arrest at G0/G1 phase of bladder cancer cells. International journal of oncology 50, 263-271 (2017).

40. Inoue, J. et al. Lysosomal-associated protein multispanning transmembrane 5 gene (LAPTM5) is associated with spontaneous regression of neuroblastomas. PloS one 4, e7099 (2009).

41. Seker, F. et al. Identification of SERPINE1 as a Regulator of Glioblastoma Cell Dispersal with Transcriptome Profiling. Cancers 11 (2019). 


\section{Table 1 (on next page)}

Summary of TCGA and GEO Clear Cell Renal Cell Carcinoma datasets. 
1

\begin{tabular}{lllllll}
\hline Author, year & Sample & GEO no. & Platform & Normal & Tumor & (Refs.) \\
\hline Stickel JS et al, 2009 & CCRCC & GSE12606 & GPL570 & 3 & 3 & {$[10]$} \\
\hline Gerlinger M et al, 2014 & CCRCC & GSE53000 & GPL6244 & 6 & 54 & {$[11]$} \\
\hline Thibodeau BJ et al, 2016 & CCRCC & GSE68417 & GPL6244 & 29 & 14 & {$[12]$} \\
\hline Wotschofsky Z et al, 2016 & CCRCC & GSE66272 & GPL5029 & 27 & 26 & {$[\mathbf{1 3}]$} \\
\hline TCGA & CCRCC & - & - & 72 & 539 & \\
\hline
\end{tabular}

2 TCGA, The Cancer Genome Atlas. GEO, Gene Expression Omnibus 3 
Table 2 (on next page)

GO and KEGG analysis of differentially expressed DEG associated with CCRCC. 
2 Table 2 GO and KEGG analysis of up-and down-regulated DEGs associated with CCRCC

\begin{tabular}{|c|c|c|c|c|}
\hline Expression & Category & Term & Count & P-value \\
\hline \multirow[t]{17}{*}{ Upregulated } & GO_BP & GO:0006954 inflammatory response & 30 & $1.30 \mathrm{E}-12$ \\
\hline & GO_BP & GO:0007165 signal transduction & 29 & $1.31 \mathrm{E}-02$ \\
\hline & GO_BP & GO:0007155 cell adhesion & 26 & 5.33E-08 \\
\hline & GO_BP & GO:0006955 immune response & 24 & $1.76 \mathrm{E}-07$ \\
\hline & GO_BP & GO:0042493 response to drug & 21 & $5.92 \mathrm{E}-08$ \\
\hline & GO_BP & GO:0045087 innate immune response & 19 & $1.36 \mathrm{E}-04$ \\
\hline & GO_BP & GO:0030198 extracellular matrix organization & 18 & $1.08 \mathrm{E}-08$ \\
\hline & GO_BP & GO:0050900 leukocyte migration & 17 & $6.55 \mathrm{E}-11$ \\
\hline & GO_BP & GO:0001525 angiogenesis & 17 & $4.06 \mathrm{E}-07$ \\
\hline & GO_BP & GO:0008284 positive regulation of cell proliferation & 17 & $2.57 \mathrm{E}-03$ \\
\hline & GO_CC & GO:0005886 plasma membrane & 108 & $1.13 \mathrm{E}-09$ \\
\hline & GO_CC & GO:0016021 integral component of membrane & 107 & $2.03 \mathrm{E}-04$ \\
\hline & GO_CC & GO:0070062 extracellular exosome & 66 & $2.68 \mathrm{E}-04$ \\
\hline & GO_CC & GO:0016020 membrane & 64 & $4.11 \mathrm{E}-07$ \\
\hline & GO_CC & GO:0005887 integral component of plasma membrane & 62 & $6.07 \mathrm{E}-14$ \\
\hline & GO_CC & GO:0005576 extracellular region & 50 & $1.98 \mathrm{E}-06$ \\
\hline & GO_CC & GO:0005615 extracellular space & 49 & $2.48 \mathrm{E}-08$ \\
\hline
\end{tabular}




\begin{tabular}{|c|c|c|c|}
\hline GO_CC & GO:0009986 cell surface & 31 & $1.08 \mathrm{E}-09$ \\
\hline GO_CC & GO:0005783 endoplasmic reticulum & 20 & 4.86E-02 \\
\hline GO_CC & GO:0000139 Golgi membrane & 17 & $1.94 \mathrm{E}-02$ \\
\hline GO_MF & GO:0005515 protein binding & 154 & $6.05 \mathrm{E}-03$ \\
\hline GO_MF & GO:0005524 ATP binding & 36 & $6.15 \mathrm{E}-03$ \\
\hline GO_MF & GO:0042803 protein homodimerization activity & 21 & $7.66 \mathrm{E}-03$ \\
\hline GO_MF & GO:0042802 identical protein binding & 20 & $1.92 \mathrm{E}-02$ \\
\hline GO_MF & GO:0005102 receptor binding & 19 & $7.41 \mathrm{E}-06$ \\
\hline GO_MF & GO:0004872 receptor activity & 14 & $2.70 \mathrm{E}-05$ \\
\hline GO_MF & GO:0004672 protein kinase activity & 12 & $2.09 \mathrm{E}-02$ \\
\hline GO_MF & GO:0005215 transporter activity & 8 & $3.41 \mathrm{E}-02$ \\
\hline GO_MF & GO:0004888 transmembrane signaling receptor activity & 8 & 4.43E-02 \\
\hline GO_MF & GO:0005201 extracellular matrix structural constituent & 7 & 5.17E-04 \\
\hline KEGG & hsa04151: PI3K-Akt signaling pathway & 19 & $2.01 \mathrm{E}-04$ \\
\hline KEGG & hsa04510: Focal adhesion & 17 & $3.76 \mathrm{E}-06$ \\
\hline KEGG & hsa05200: Pathways in cancer & 17 & $5.96 \mathrm{E}-03$ \\
\hline KEGG & hsa04066: HIF-1 signaling pathway & 12 & 3.37E-06 \\
\hline KEGG & hsa04015: Rap1 signaling pathway & 12 & $3.56 \mathrm{E}-03$ \\
\hline KEGG & hsa04145: Phagosome & 10 & $3.39 \mathrm{E}-03$ \\
\hline
\end{tabular}




\begin{tabular}{|c|c|c|c|c|}
\hline & KEGG & hsa05205: Proteoglycans in cancer & 10 & $2.05 \mathrm{E}-02$ \\
\hline & KEGG & hsa05150: Staphylococcus aureus infection & 9 & $1.14 \mathrm{E}-05$ \\
\hline & KEGG & hsa04611: Platelet activation & 9 & $4.86 \mathrm{E}-03$ \\
\hline & KEGG & hsa05133: Pertussis & 8 & 7.79E-04 \\
\hline \multirow[t]{15}{*}{ Downregulated } & GO_BP & GO:0055114 oxidation-reduction process & 33 & $3.33 \mathrm{E}-05$ \\
\hline & GO_BP & GO:0034220 ion transmembrane transport & 19 & $5.29 \mathrm{E}-06$ \\
\hline & GO_BP & GO:0042493 response to drug & 18 & $1.62 \mathrm{E}-03$ \\
\hline & GO_BP & GO:0006811 ion transport & 14 & $1.66 \mathrm{E}-05$ \\
\hline & GO_BP & GO:0055085 transmembrane transport & 14 & $8.16 \mathrm{E}-03$ \\
\hline & GO_BP & GO:0007588 excretion & 13 & 4.97E-11 \\
\hline & GO_BP & GO:0010628 positive regulation of gene expression & 13 & $3.13 \mathrm{E}-02$ \\
\hline & GO_BP & GO:0006814 sodium ion transport & 12 & 4.94E-06 \\
\hline & GO_BP & GO:0001822 kidney development & 12 & $8.92 \mathrm{E}-06$ \\
\hline & GO_BP & GO:0008152 metabolic process & 12 & $3.22 \mathrm{E}-03$ \\
\hline & GO_CC & GO:0016021 integral component of membrane & 171 & 4.63E-07 \\
\hline & GO_CC & GO:0070062 extracellular exosome & 170 & $1.01 \mathrm{E}-33$ \\
\hline & GO_CC & GO:0005886 plasma membrane & 162 & $2.99 \mathrm{E}-12$ \\
\hline & GO_CC & GO:0005887 integral component of plasma membrane & 76 & $3.16 \mathrm{E}-11$ \\
\hline & GO_CC & GO:0005615 extracellular space & 50 & $1.87 \mathrm{E}-03$ \\
\hline
\end{tabular}




\begin{tabular}{|c|c|c|c|}
\hline GO_CC & GO:0016324 apical plasma membrane & 44 & $4.39 \mathrm{E}-22$ \\
\hline GO_CC & GO:0016323 basolateral plasma membrane & 28 & $2.05 \mathrm{E}-14$ \\
\hline GO_CC & GO:0009986 cell surface & 24 & $5.53 \mathrm{E}-03$ \\
\hline GO_CC & GO:0005759 mitochondrial matrix & 19 & 8.74E-04 \\
\hline GO_CC & GO:0043025 neuronal cell body & 15 & $1.88 \mathrm{E}-02$ \\
\hline GO_MF & GO:0042803 protein homodimerization activity & 33 & 7.43E-04 \\
\hline GO_MF & GO:0005509 calcium ion binding & 29 & 7.77E-03 \\
\hline GO_MF & GO:0043565 sequence-specific DNA binding & 20 & 4.43E-02 \\
\hline GO_MF & GO:0016491 oxidoreductase activity & 13 & $3.24 \mathrm{E}-03$ \\
\hline GO_MF & GO:0046983 protein dimerization activity & 11 & $3.31 \mathrm{E}-03$ \\
\hline GO_MF & GO:0008201 heparin binding & 11 & $5.22 \mathrm{E}-03$ \\
\hline GO_MF & GO:0005088 Ras guanyl-nucleotide exchange factor activity & 10 & $1.78 \mathrm{E}-03$ \\
\hline GO_MF & GO:0003824 catalytic activity & 10 & 3.74E-02 \\
\hline GO_MF & GO:0016787 hydrolase activity & 10 & 4.07E-02 \\
\hline GO_MF & GO:0046934 phosphatidylinositol-4,5-bisphosphate 3-kinase activity & 9 & $1.10 \mathrm{E}-04$ \\
\hline KEGG & hsa01100: Metabolic pathways & 68 & $8.56 \mathrm{E}-08$ \\
\hline KEGG & hsa01130: Biosynthesis of antibiotics & 19 & 5.17E-05 \\
\hline KEGG & hsa01200: Carbon metabolism & 11 & $1.83 \mathrm{E}-03$ \\
\hline KEGG & hsa04960: Aldosterone-regulated sodium reabsorption & 9 & $1.45 \mathrm{E}-05$ \\
\hline
\end{tabular}




\begin{tabular}{llll}
\hline KEGG & hsa04978: Mineral absorption & 9 & $3.69 \mathrm{E}-05$ \\
KEGG & hsa00010: Glycolysis / Gluconeogenesis & 9 & $7.57 \mathrm{E}-04$ \\
KEGG & hsa04610: Complement and coagulation cascades & 8 & $4.14 \mathrm{E}-03$ \\
KEGG & hsa04966: Collecting duct acid secretion & 7 & $1.11 \mathrm{E}-04$ \\
KEGG & hsa00260: Glycine, serine and threonine metabolism & 7 & $9.10 \mathrm{E}-04$ \\
KEGG & hsa00280: Valine, leucine and isoleucine degradation & 7 & $2.46 \mathrm{E}-03$ \\
\hline
\end{tabular}

3 
Table 3 (on next page)

The top 35 DEGs identified with the degree in PPI network. 
1 Table 3. The top 35 DEGs identified with the degree in PPI network. (Degree $\geq 37$ )

\begin{tabular}{|c|c|c|c|c|}
\hline Expression & Genes & Degree & Genes & Degree \\
\hline \multirow[t]{15}{*}{ Upregulated } & EGFR & 110 & C3AR1 & 52 \\
\hline & VEGFA & 100 & CYBB & 51 \\
\hline & PTPRC & 93 & $\mathrm{LCP} 2$ & 50 \\
\hline & FN1 & 90 & TLR7 & 49 \\
\hline & ITGB2 & 70 & $\mathrm{C} 3$ & 45 \\
\hline & TLR2 & 67 & CCL5 & 44 \\
\hline & MMP9 & 66 & TIMP1 & 43 \\
\hline & CD86 & 64 & HCK & 43 \\
\hline & CXCR4 & 62 & FCGR2A & 42 \\
\hline & ICAM1 & 62 & CD53 & 41 \\
\hline & CCND1 & 61 & CTSS & 41 \\
\hline & TYROBP & 59 & LAPTM5 & 40 \\
\hline & CSF1R & 58 & LOX & 38 \\
\hline & PLEK & 55 & SERPINE1 & 37 \\
\hline & ITGAX & 53 & CAV1 & 37 \\
\hline \multirow[t]{3}{*}{ Downregulated } & ALB & 134 & KNG1 & 52 \\
\hline & EGF & 80 & KIT & 44 \\
\hline & ERBB2 & 67 & & \\
\hline
\end{tabular}

2

3

4

5

6

7

8

9 
Figure 1

Flow chart of data collection, processing, analysis and verification in this study.

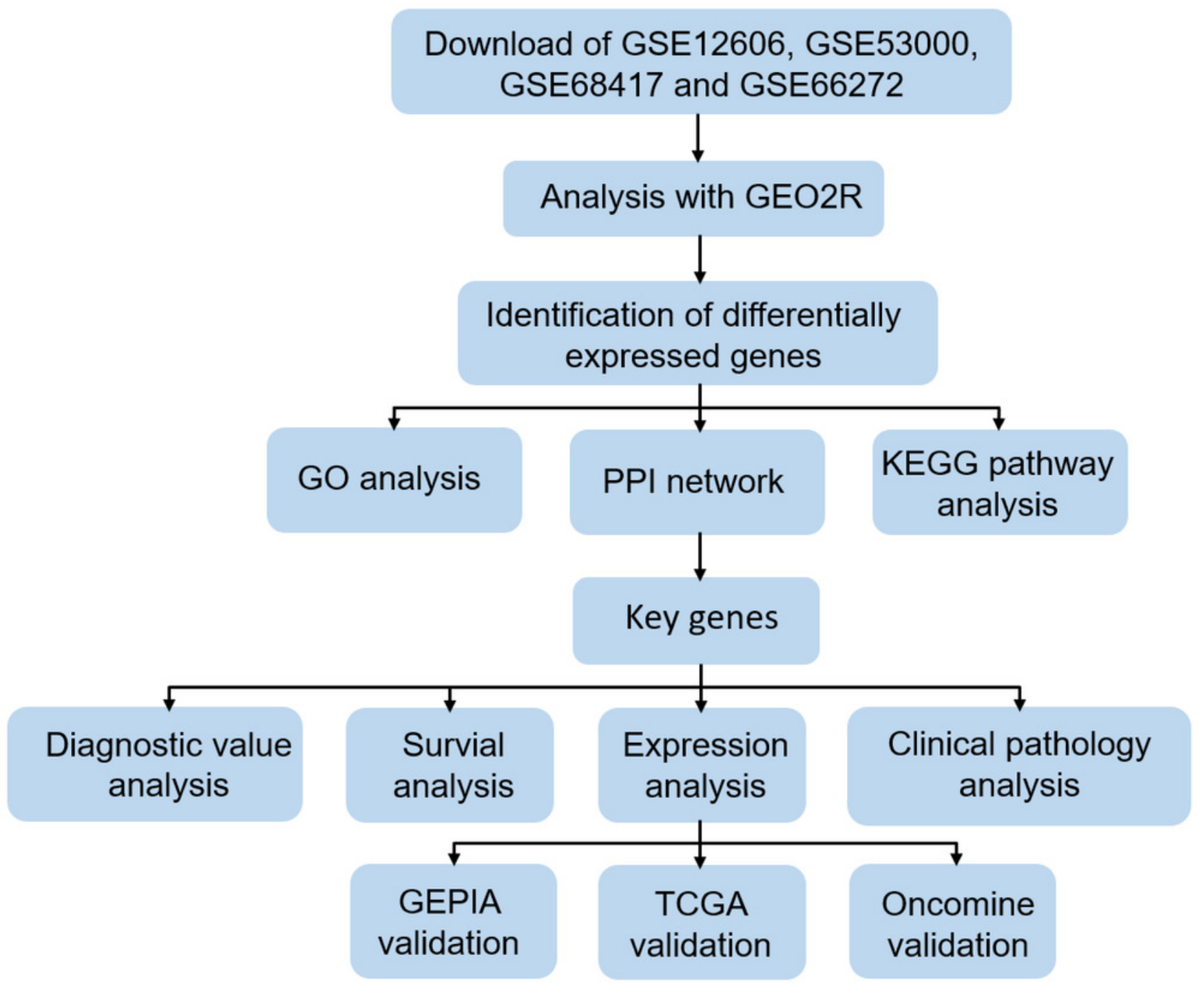


Figure 2

Screening of differentially expressed genes.

(A-D) The volcano plot of all DEGs respectively in GSE12606, GSE53000, GSE68417, and GSE66272 datasets. Red and green nodes represent up-regulated genes and down-regulated genes, respectively. (E-F)738 DEGs were identified in four profile datasets (GSE12606, GSE53000, GSE68417, and GSE66272), 289 upregulated genes, 449 downregulated genes. 
A

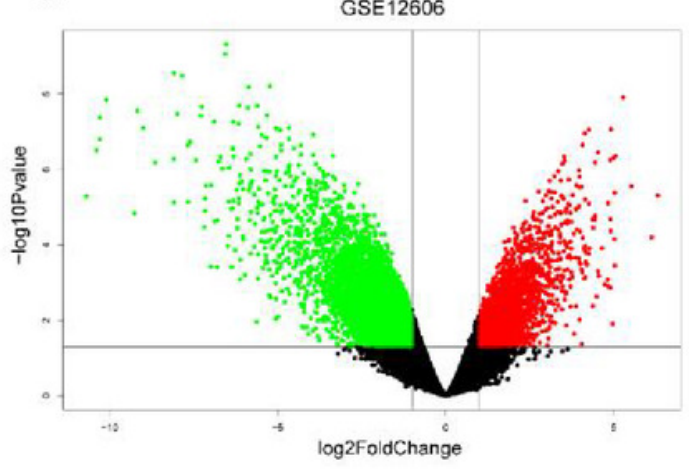

C

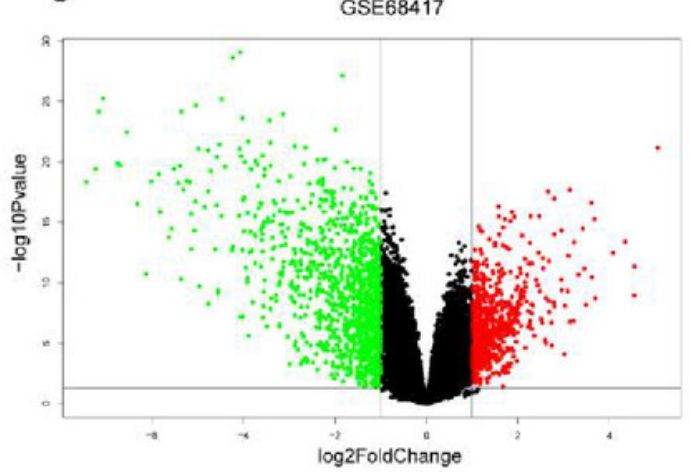

E

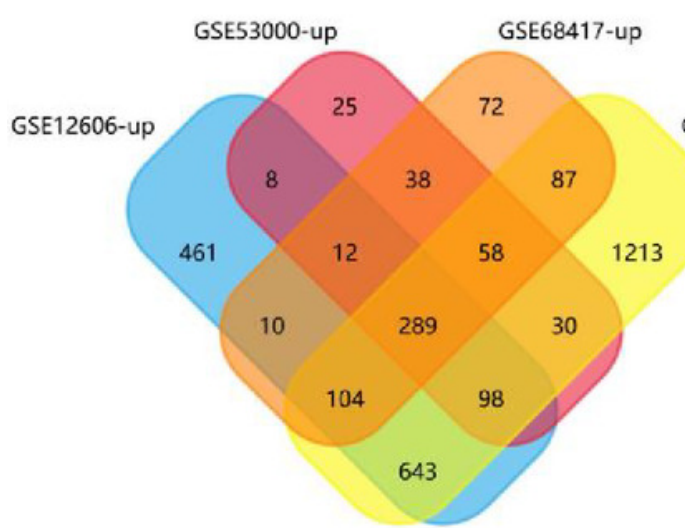

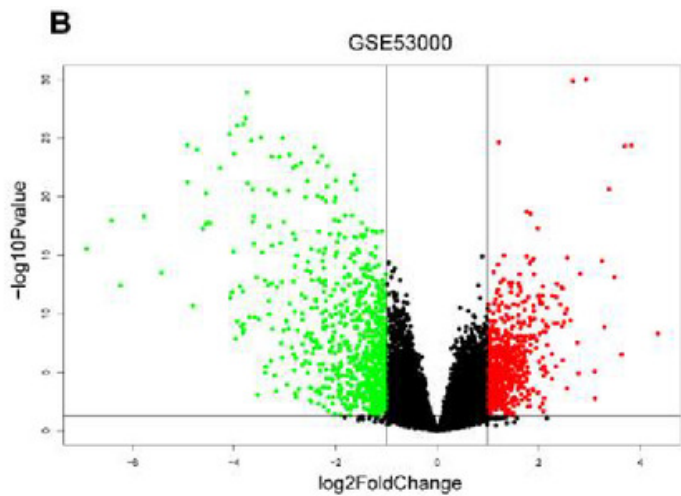

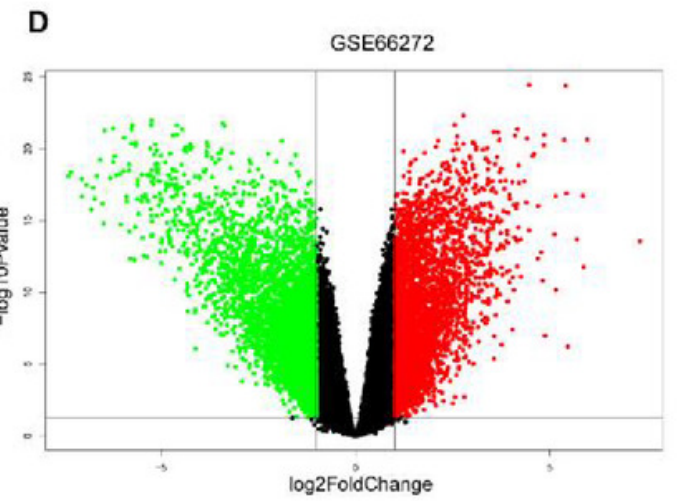

$\mathbf{F}$

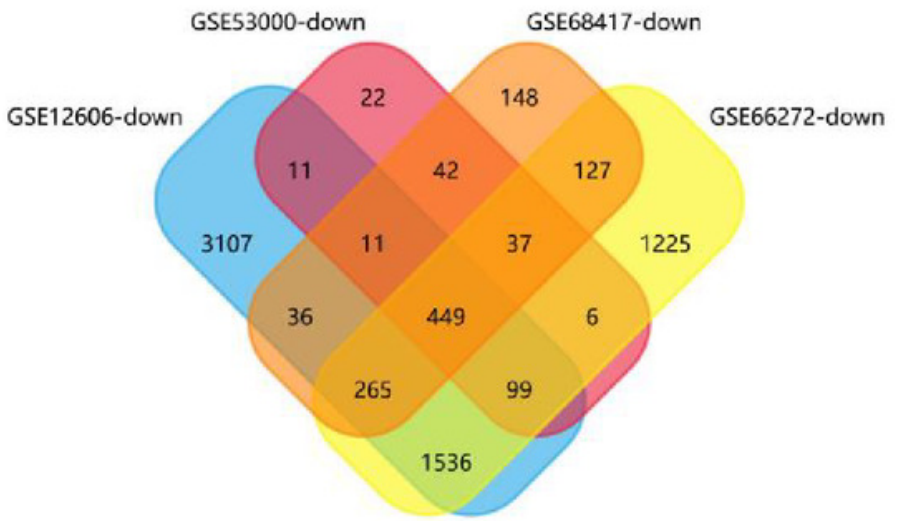




\section{Figure 3}

Enrichment analysis of GO and KEGG with up-regulated DEG.

(A) The biological process of $\mathrm{GO}$ analysis showed that the up-regulation of DEGs was mainly related to cell adhesion, inflammatory response, signal transduction, and immune response.

(B) The enrichment analysis of up-regulated DEGs cell components is mainly related to the cellular exosomes, integral component of membrane, plasma membrane, and integral component of plasma membrane. (C) The molecular function of GO analysis showed that the up-regulation of DEGs was mainly related to protein binding, identical protein bind, ATP binding capacity, and protein homodimerization activity. (D) The KEGG pathways related to the up-regulation of DEGs expression mainly include the PI3K-Akt signaling pathway, focal adhesion, pathways in cancer, and HIF-1 signaling pathway. (E) The biological process of GO analysis showed that the downregulation of DEGs was mainly related to oxidation-reduction process proteolysis, ion transmembrane transport, response to the drug, and ion transport. (F) The enrichment analysis of down-regulated DEGs cell components is mainly related to integral components of membrane, plasma membrane, cellular exosomes, and integral component of plasma membrane. $(\mathrm{G})$ The molecular function of $\mathrm{GO}$ analysis showed that the downregulation of DEGs was mainly related to protein homodimerization activity, calcium ion binding, oxidoreductase activity, and sequence-specific DNA binding. (H) The KEGG pathways related to the down-regulation of DEGs expression mainly include metabolic pathway, Biosynthesis of antibiotics, Carbon metabolism, and Aldosterone-regulated sodium reabsorption. 
A

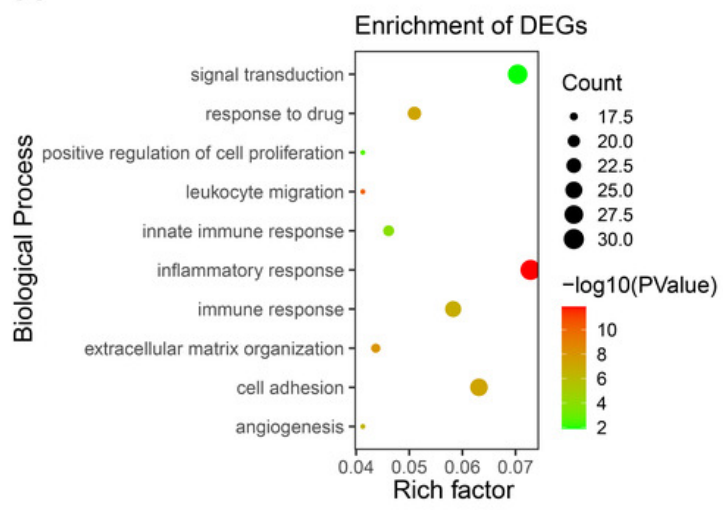

C

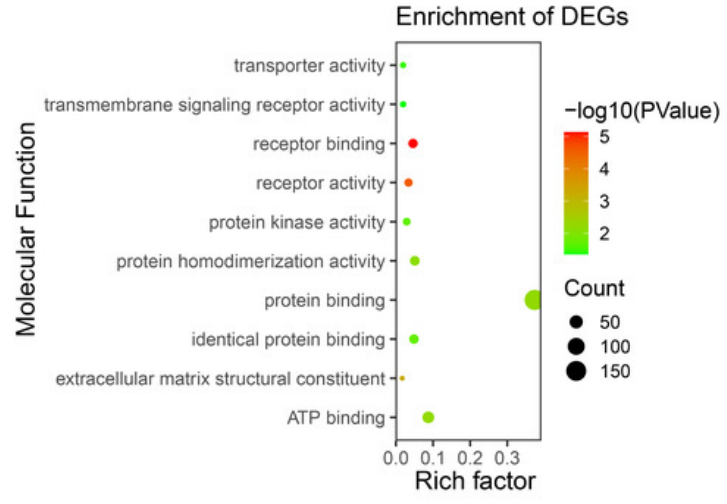

E

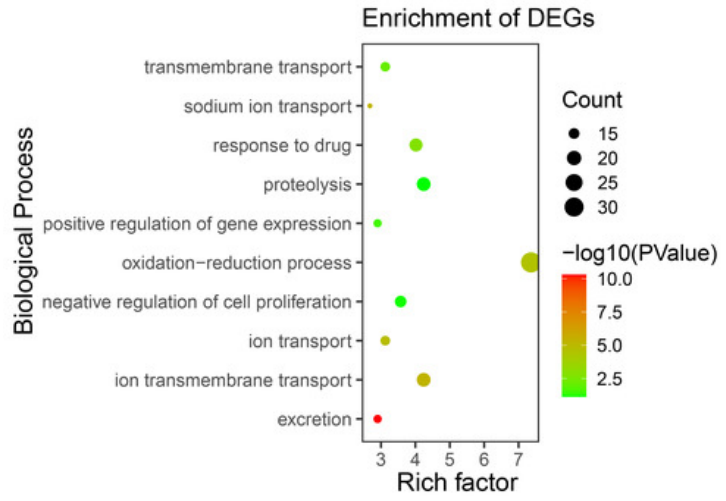

G

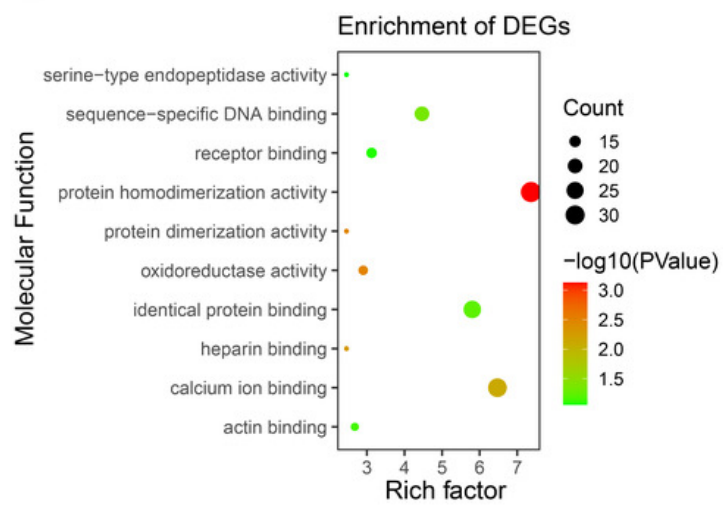

B

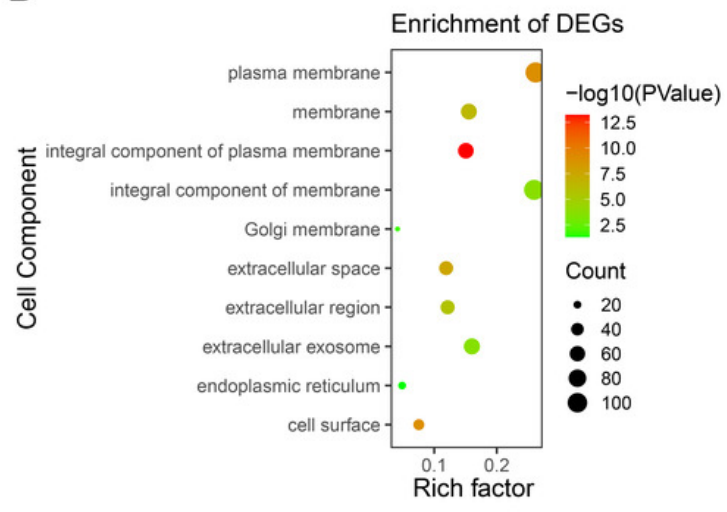

D

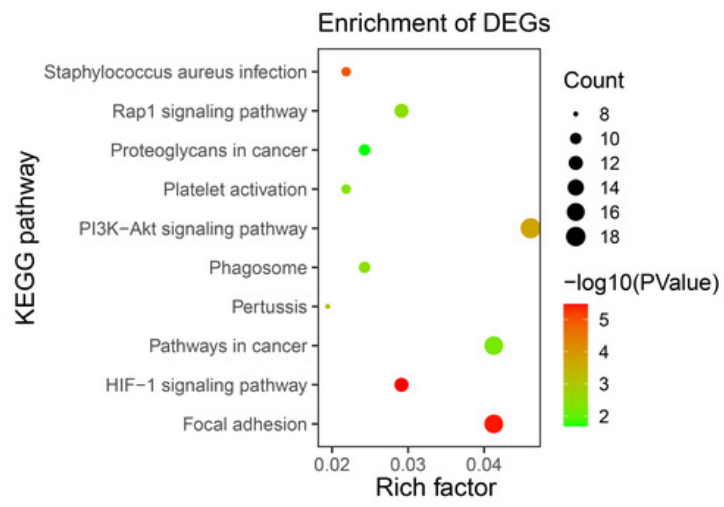

$\mathbf{F}$

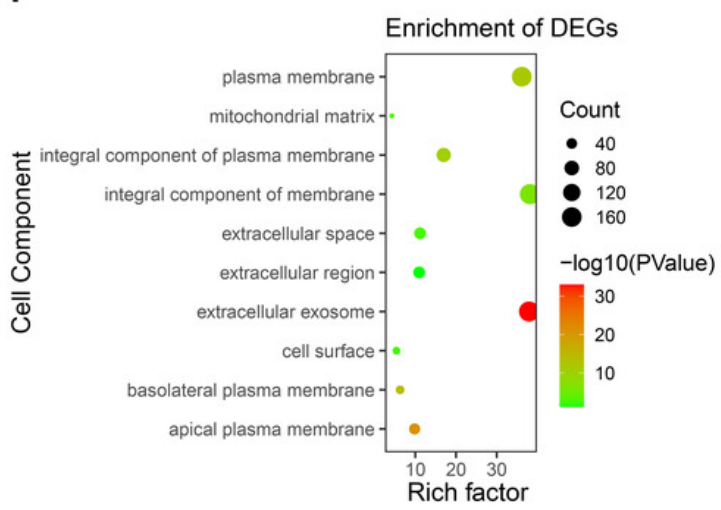

H

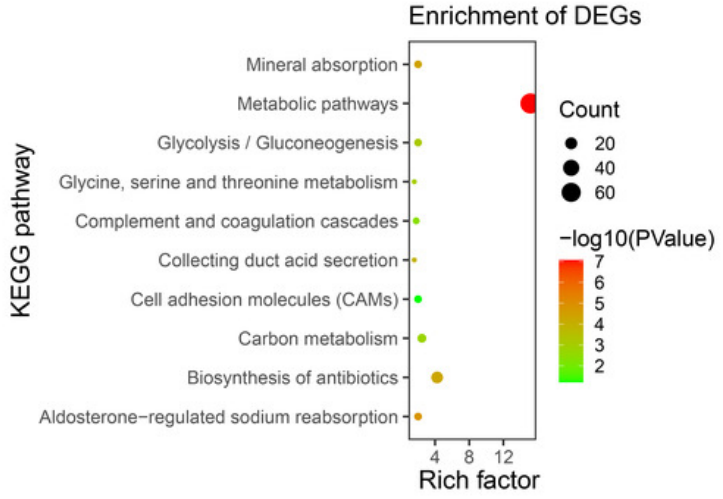




\section{Figure 4}

PPI network and GO enrichment analysis of key genes.

(A) The PPI network is constructed, including up-regulated genes and down-regulated genes, and considers that the confidence level $\geq 0.4$ is significant for PPI. (B-C) GO enrichment analysis of all DEGs in PPI network. (D) PPI network of the top 35 DEGs according to the degree (degree $\geq 37$ ). (E) GO enrichment analysis of the top 35 DEGs. 
A

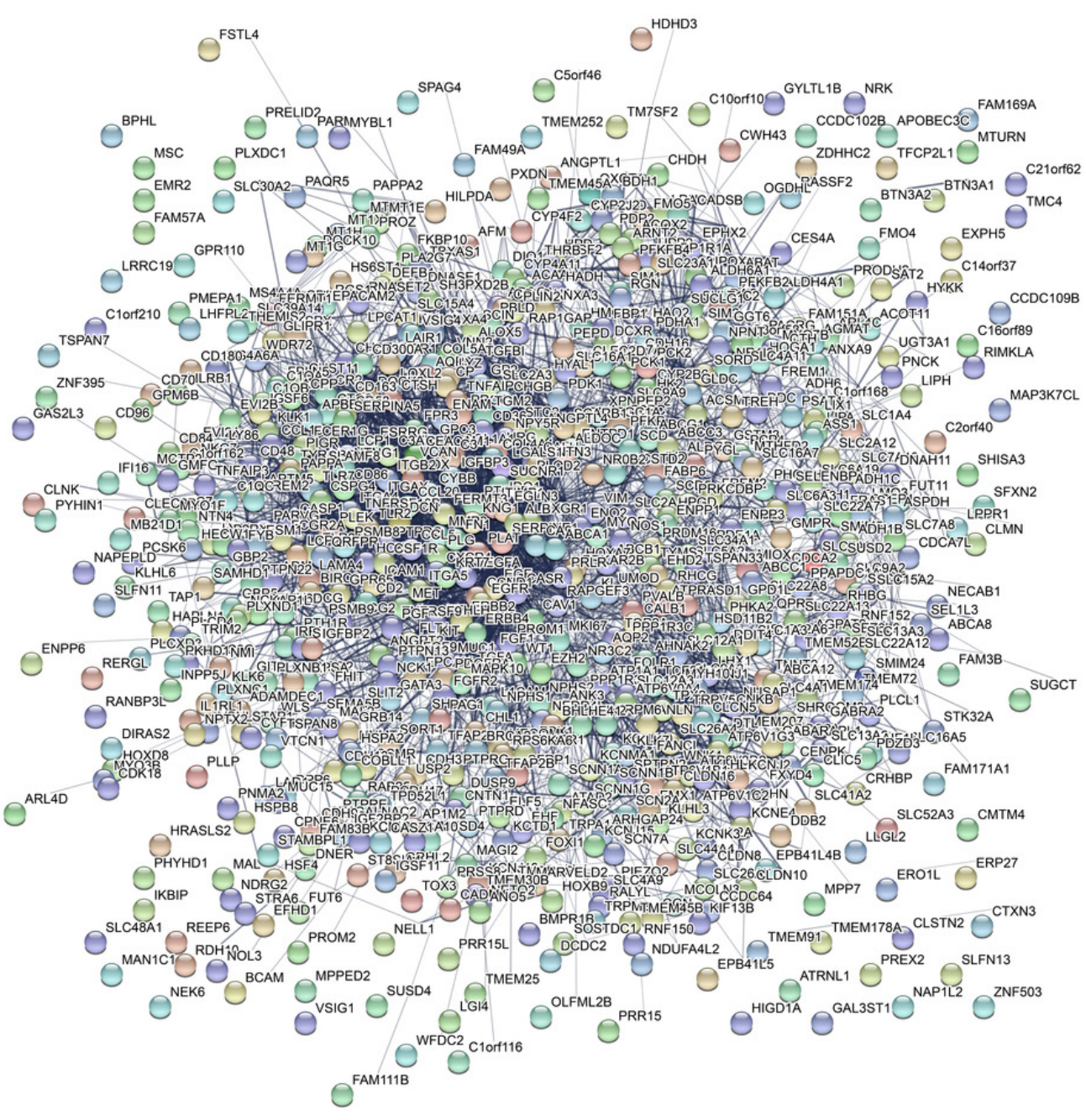

B

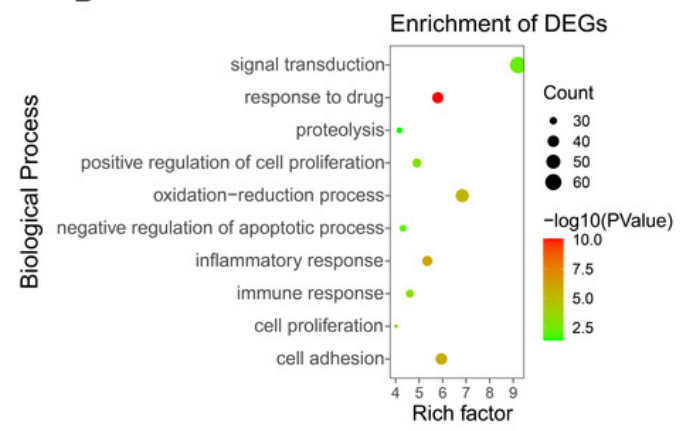

C

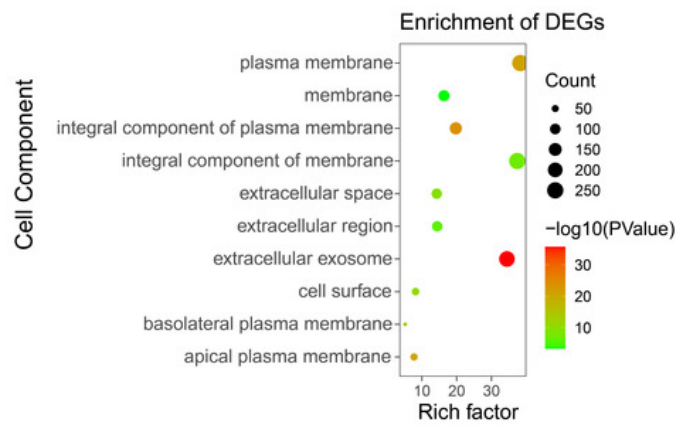

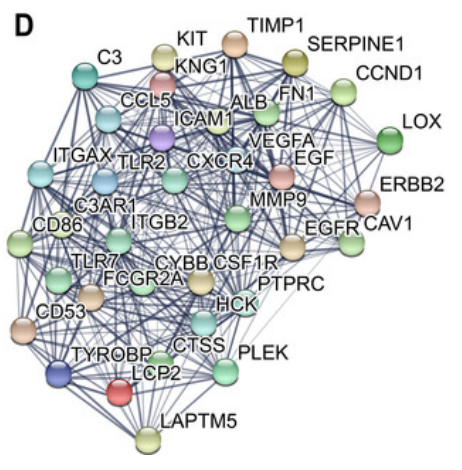

E

GO enrichment analysis of the top 35 DEGs.

\begin{tabular}{lcc}
\hline Term & Count $(\geq 5)$ & P-value \\
\hline GO-CC: 0005615 extracellular space & 11 & $2.531 \mathrm{E}-06$ \\
GO-CC: 0070062 extracellular exosome & 11 & $1.313 \mathrm{E}-03$ \\
GO-BP: 0006954 inflammatory response & 6 & $8.250 \mathrm{E}-05$ \\
GO-CC: 0005887 integral component of plasma membrane & 6 & $1.554 \mathrm{E}-02$ \\
GO-CC: 0009986 cell surface & 5 & $2.203 \mathrm{E}-03$ \\
\hline
\end{tabular}




\section{Figure 5}

Key gene expression between normal kidney and CCRCC tissues.

(A-C) Box plot showing the expression of ITGAX, LAPTM5, SERPINE1 in GEPIA database. These three genes are highly expressed in CCRCC. (D-F) In the TCGA database, compared with adjacent normal tissues, the expression of ITGAX, LAPTM5, SERPINE1 mRNA in 72 pairs of CCRCC tissues increased significantly. (G-I) The expression of ITGAX, LAPTM5, SERPINE1 are significantly increased in Oncomine Gumz renal database. ${ }^{*} \mathrm{P}<0.05$, ${ }^{* * \mathrm{P}}<0.01$, ${ }^{* * * P}<$ 0.001. (J-L)The box plot showing the promoter methylation levels of ITGAX, LAPTM5 and SERPINE1 in the UALCAN database. $P<0.01$. 
A

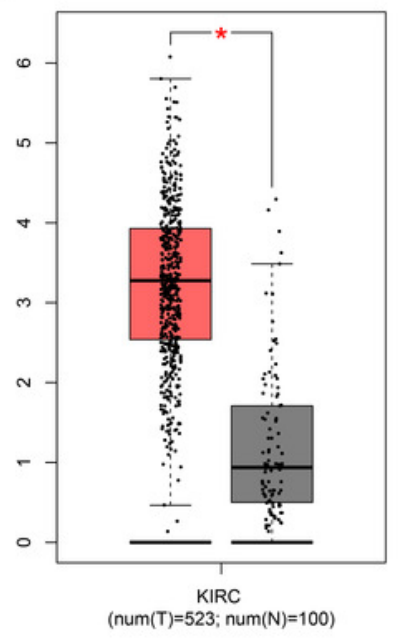

D

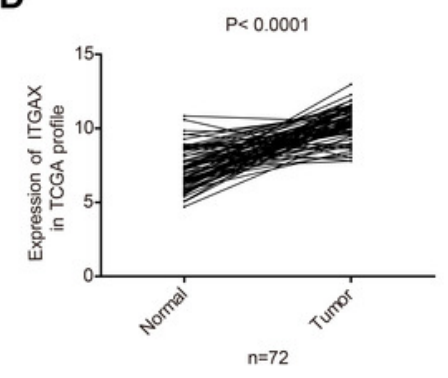

G

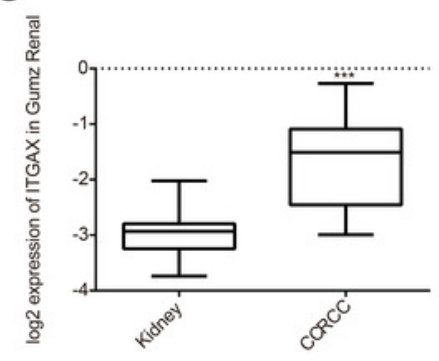

J

Promoter methylation level of ITGAX in KIRC

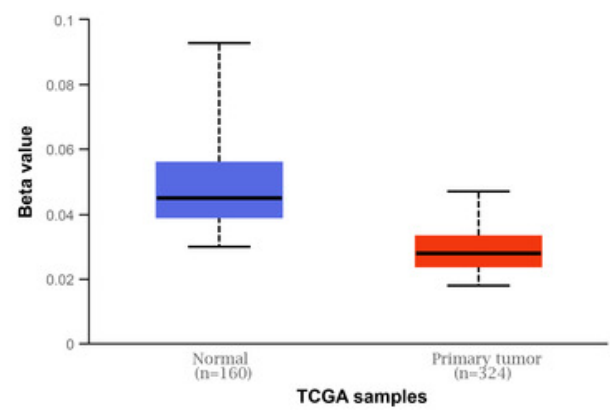

B

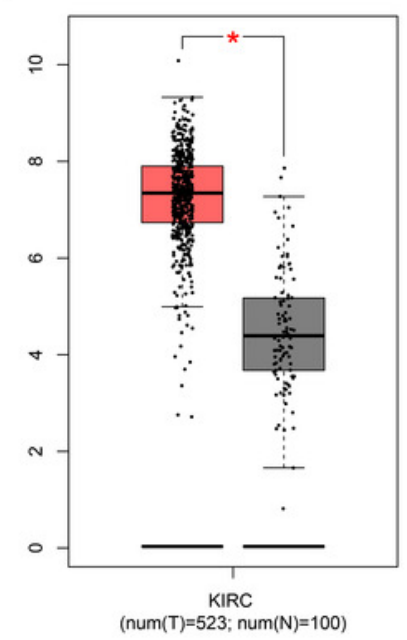

E

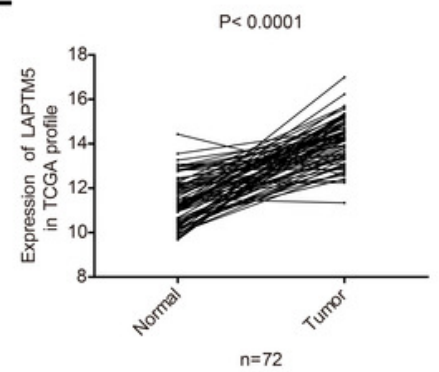

H

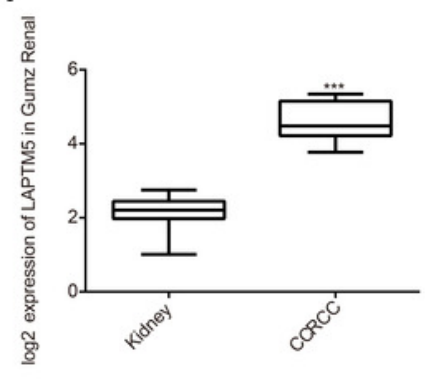

K

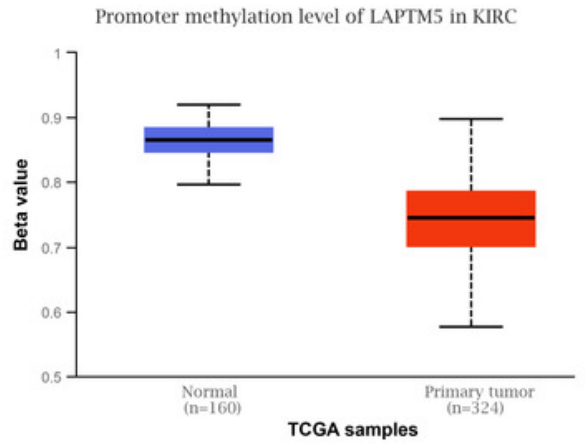

C SERPINE1

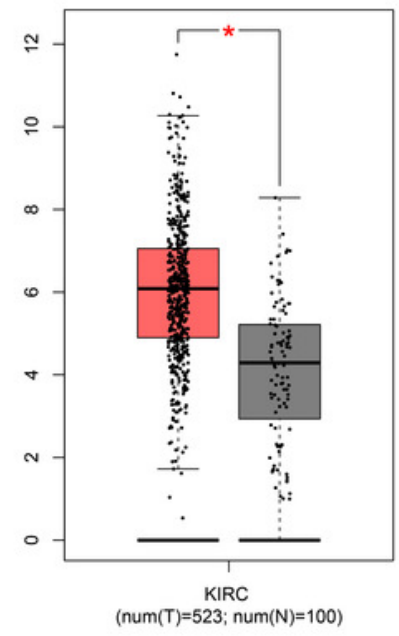

F

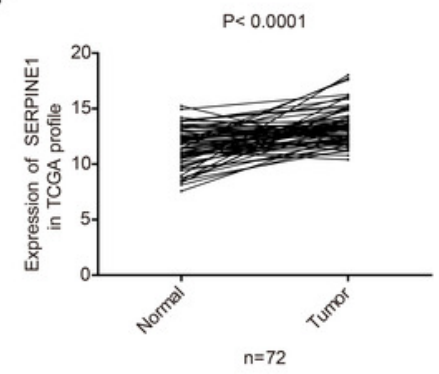

I

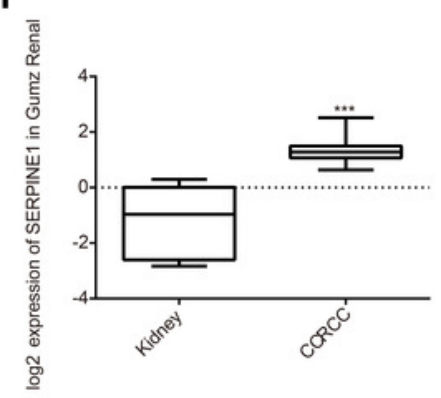

L

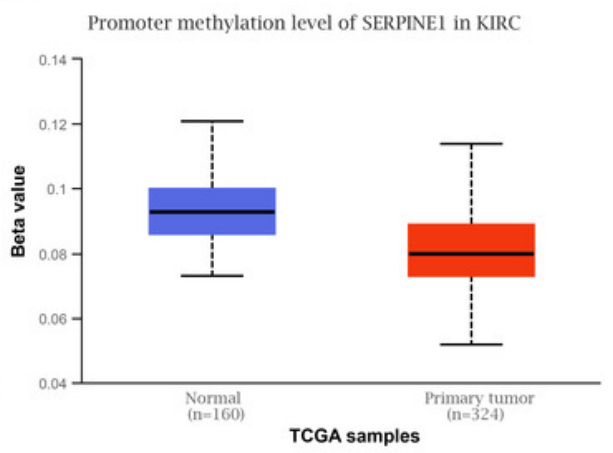


Figure 6

The relationship between ITGAX, LAPTM5, and SERPINE1 expression and clinical pathology of CCRCC.

(A-C) Boxplot showing that ITGAX, LAPTM5, and SERPINE1 mRNA expression were significantly related to pathological grades, and patients in grade 4 have the highest expression in CCRCC according to UALCAN databases. (D-F) Boxplot showing that the expression of ITGAX, LAPTM5, and SERPINE1 mRNA in CCRCC samples are significantly correlated with severe clinical staging and the mRNA expression of ITGAX, LAPTM5, and SERPINE1 were higher in patients with stage 4 according to UALCAN databases. $* P<0.05$, $* * \mathrm{P}<0.01, * * * \mathrm{P}<0.001$.

A

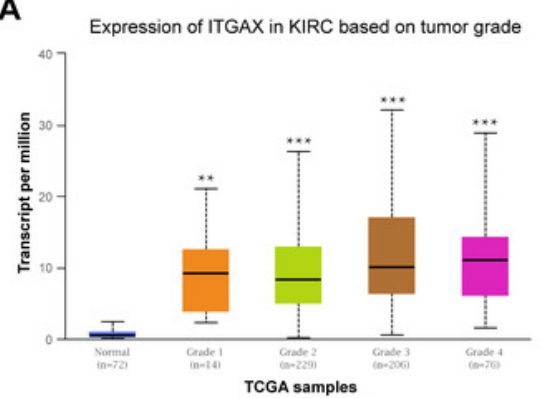

D

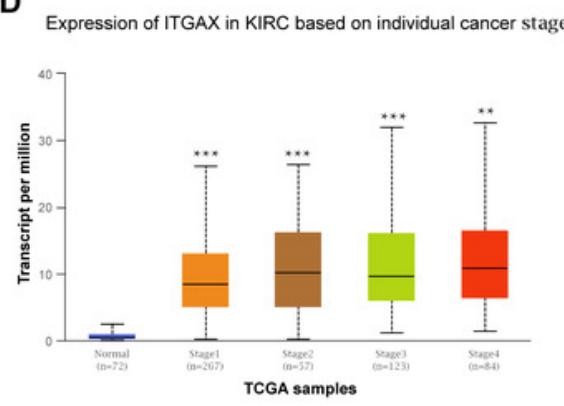

B

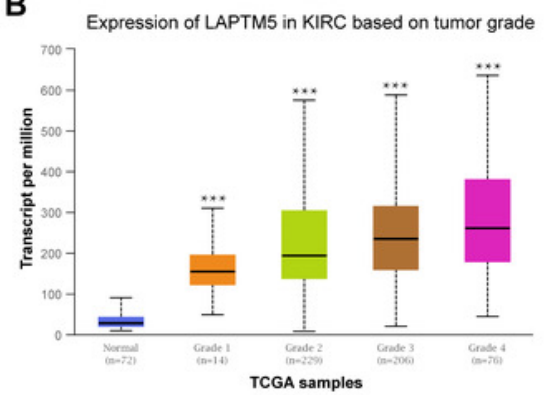

E

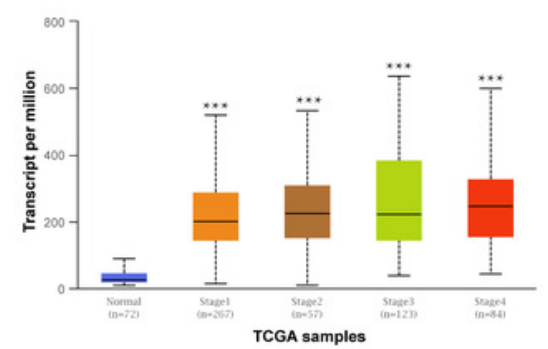

C

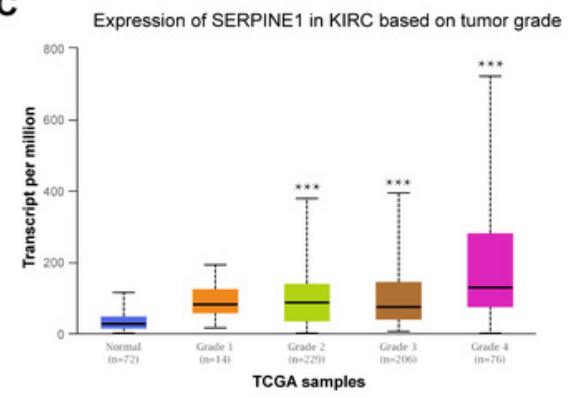

F

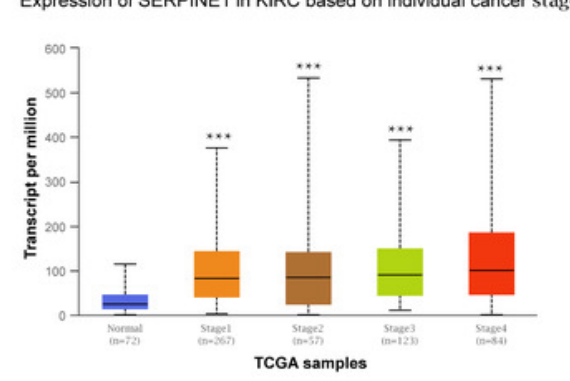


Figure 7

Survival and diagnostic value of ITGAX, LAPTM5, and SERPINE1 in CCRCC

(A-C) The overall survival of ITGAX, LAPTM5, and SERPINE1.The results showed that the high expression of three key genes in CCRCC was negatively correlated with prognosis. (D) ROC curve of ITGAX ( $A \cup C=96.315$, cutoff value=8.836, Sensitivity=92.579 Specificity=91.667).

(E) ROC curve of LAPTM5, (AUC $=94.808$, cutoff value $=12.983$ Sensitivity $=85.158$

Specificity=94.444). (F) ROC curve of SERPINE1, AUC $=76.272$, cutoff value $=12,646$, Sensitivity $=66.234$ Specificity $=75$ ).
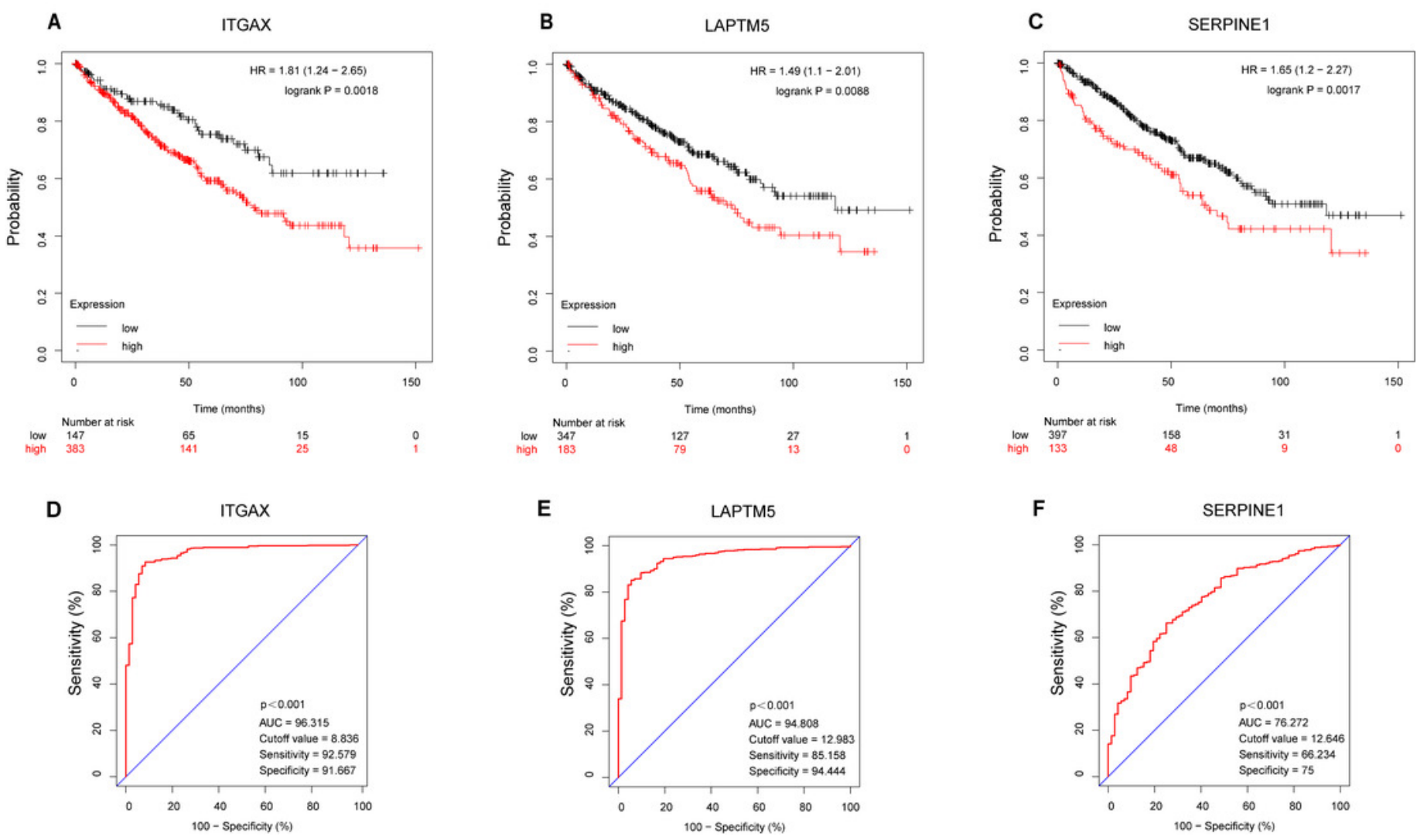